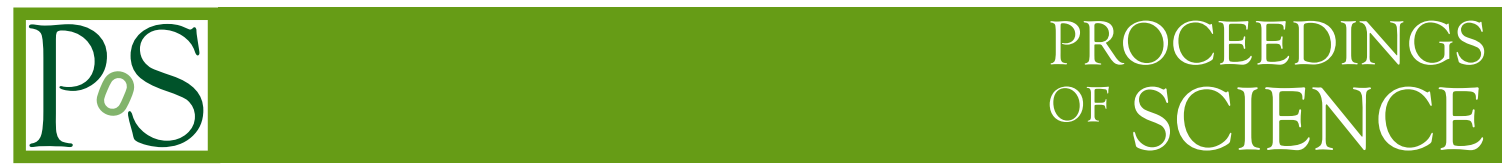

\title{
New measurements in the quarkonium sector with focus on new discriminatory observables
}

\section{Minjung $\mathrm{Kim}^{a, *}$ for the ALICE, ATLAS, CMS and LHCb Collaborations}

${ }^{a}$ Physikalisches Institut, Ruprecht-Karls-Universität Heidelberg,

Im Neuenheimer Feld 226, Heidelberg, Germany

E-mail: minjung.kim@cern.ch

Measurements of quarkonium production are valuable probes of the hot and dense medium created in heavy-ion collisions. The large data sample collected at the LHC provides a wealth of quarkonium measurements including a multitude of new observables. We report on the latest measurements of quarkonium production at the LHC focusing on new observables.

The Ninth Annual Conference on Large Hadron Collider Physics - LHCP2021

7-12 June 2021

Online

* Speaker 
The higher collision energy at the LHC with respect to previous collision programs at SPS and RHIC opened new perspectives on quarkonium measurements due to the increased heavy quark production cross section. The most intuitive observable quantifying the modification of the particle production in heavy-ion collisions with respect to proton-proton (pp) collisions is the nuclear modification factor $R_{\mathrm{AA}}$, the ratio of the production in nucleus-nucleus collisions to that in $\mathrm{pp}$ collisions at the same energy scaled by the number of binary collisions. The $R_{\mathrm{AA}}$ of $J / \psi$ mesons measured in $\mathrm{Pb}-\mathrm{Pb}$ collisions at LHC [1] compared with theoretical models indicates a substantial contribution of charmonium produced at low transverse momentum $\left(p_{\mathrm{T}}\right)$ by recombination of abundantly produced $c$ and $\bar{c}$ quarks, either throughout the evolution of the quark-gluon plasma (QGP) [2] or at hadronization [3]. Meanwhile, bottomonium production study has become a strong tool to probe the full space-time evolution of the QGP as the three S-wave states of bottomonium, $\Upsilon(1 S), \Upsilon(2 S)$, and $\Upsilon(3 S)$ became accessible and their production is expected to be less affected by recombination. The measured $R_{\mathrm{AA}}$ of $\Upsilon(\mathrm{nS})$ shows a clear ordering of the suppression, $R_{\mathrm{AA}}$ $(\Upsilon(1 \mathrm{~S}))>R_{\mathrm{AA}}(\Upsilon(2 \mathrm{~S}))>R_{\mathrm{AA}}(\Upsilon(3 \mathrm{~S}))$ [4] as expected in the sequential melting scenario [5] based on colour screening [6]. At the same time, the measured production ratios are also consistent with thermal production at the phase boundary [7]. The interpretation of the underlying physics is not trivial as the observed suppression is the subtle interplay of different mechanisms $[8,9]$. The large data samples collected at the LHC based on the excellent performance of the LHC and the four experiments (ALICE [10], ATLAS [11], CMS [12] and LHCb [13]) has enabled a multitude of further measurements which are crucial to disentangle multiple effects present in quarkonium production. In these proceedings, a selection of recent quarkonium measurements at the LHC will be discussed focusing on new observables.

Various measurements of quarkonium production in pp collisions including the production of excited states and the production associated with other particles are crucial inputs to understand the production mechanisms which involve both perturbative and non-perturbative aspects of QCD [15]. In particular, quarkonium production studied differentially in the underlying event properties can
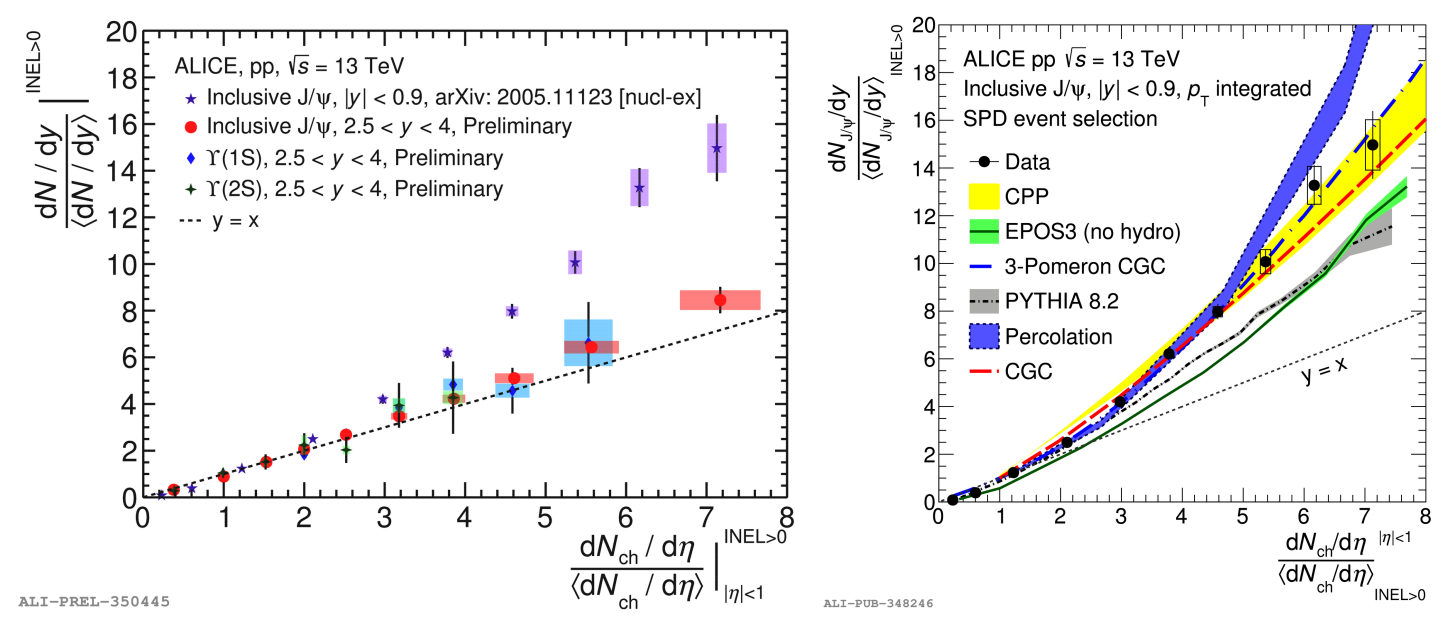

Figure 1: Relative yield of $J / \psi, \Upsilon(1 \mathrm{~S})$ and $\Upsilon(2 S)$ at forward rapidity and $J / \psi$ at midrapidity as a function of relative charged particle multiplicity at midrapidity (left) and $J / \psi$ at midrapidity together with theoretical calculations [14] (right). 

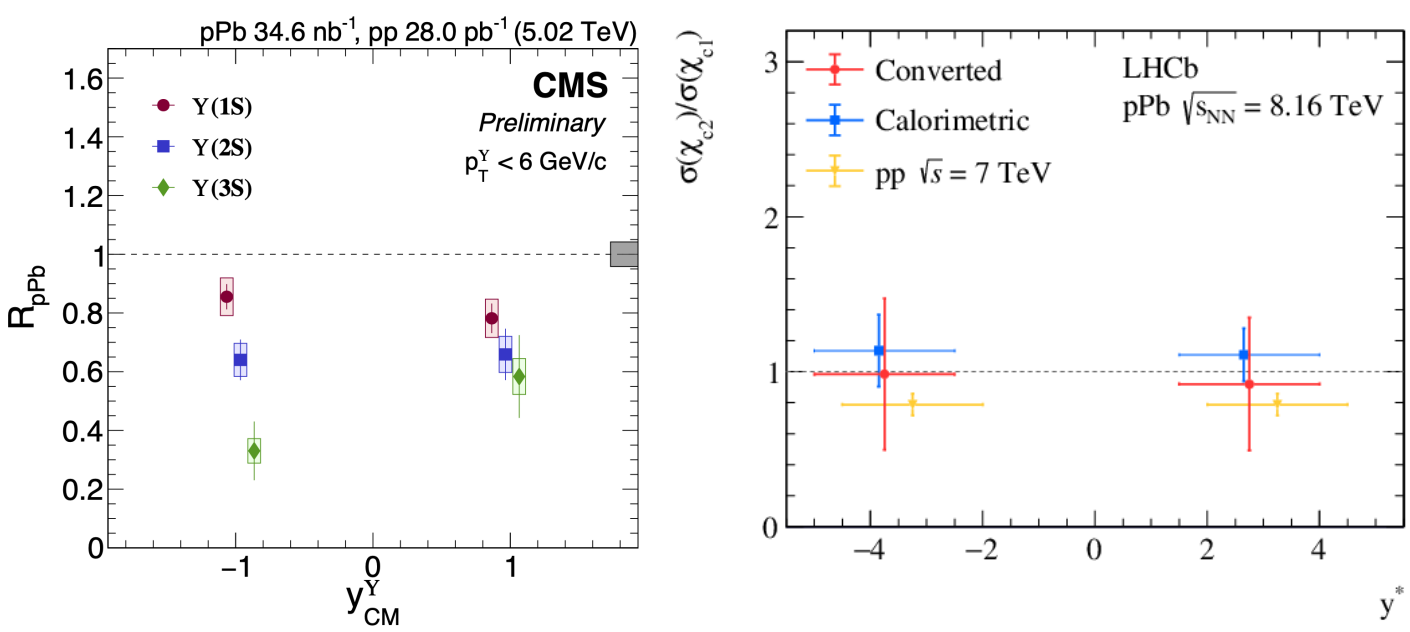

Figure 2: $R_{\mathrm{pPb}}$ of $\Upsilon(\mathrm{nS})$ [19] (left) and $\sigma\left(\chi_{\mathrm{c} 2}\right) / \sigma\left(\chi_{\mathrm{c} 1}\right)$ as a function of rapidity [21] (right).

provide insight into the interplay between hard and soft processes [16]. The multiplicity dependence of quarkonium production measured by the ALICE Collaboration is presented in Fig. 1. The linear increase with multiplicity, the latter measured at midrapidity, for $\Upsilon(1 S), \Upsilon(2 S)$ as well as $J / \psi$ at forward rapidity indicates no significant dependence on the heavy quark content and mass. The stronger increase of $J / \psi$ at midrapidity is rather well described by some models considering a reduction of the charged-particle multiplicity at high charged particle multiplicity relative to the production of $J / \psi$ from hard scatterings realized by different physics mechanisms [14]. Recently, a detailed study of the underlying event dependent $\Upsilon(\mathrm{nS})$ production was carried out by the CMS Collaboration emphasizing the need for an improved theoretical description [17].

Possible modifications of quarkonium production in the presence of a nucleus can be studied via measurements in proton-nucleus (pA) collisions which should be precisely understood to interpret the measurements in heavy-ion collisions. Since the initial-state effects for the heavy-quark pair are expected to affect similarly all different final quarkonium states, the effects leading to a break-up of the quarkonium resonances get attention as the production of excited states in various kinematic regimes becomes accessible [18]. In Fig. 2, the $R_{\mathrm{pPb}}$ values of $\Upsilon(1 \mathrm{~S}), \Upsilon(2 \mathrm{~S})$ and $\Upsilon(3 \mathrm{~S})$ measured by the CMS Collaboration are presented as a function of rapidity [19]. A clear ordering is observed in their suppression at backward rapidity, with $\Upsilon(1 \mathrm{~S})$ least suppressed and $\Upsilon(3 S)$ most suppressed similar to charmonium results in $\mathrm{p}-\mathrm{Pb}$ collisions, showing larger suppression of $\psi(2 \mathrm{~S})$ with respect to $J / \psi$ [20]. This trend cannot be described by theoretical models only including initial-state effects, suggesting the presence of final-state effects in $\mathrm{p}-\mathrm{Pb}$ collisions. The first comparison of the cross section ratio $\sigma\left(\chi_{\mathrm{c} 2}\right) / \sigma\left(\chi_{\mathrm{c} 1}\right)$ in $\mathrm{p}-\mathrm{Pb}$ collisions and in pp collisions, provided by the LHCb Collaboration as shown in Fig. 2, suggests that nuclear effects occurring in $\mathrm{p}-\mathrm{Pb}$ collisions impact similarly the $\chi_{\mathrm{c} 2}$ and $\chi_{\mathrm{c} 1}$ which have a relatively small binding energy difference [21].

Besides the $R_{\mathrm{AA}}$, elliptic flow, i.e. the second harmonic coefficient $v_{2}$ of the Fourier decomposition of the azimuthal particle distribution was measured to understand the quarkonium production mechanism in $\mathrm{Pb}-\mathrm{Pb}$ collisions as well as thermalization of charm and beauty quark. The non-zero $J / \psi v_{2}$ at low $p_{\mathrm{T}}$ measured by the ALICE Collaboration [22] shown in Fig. 3 supports a large degree 

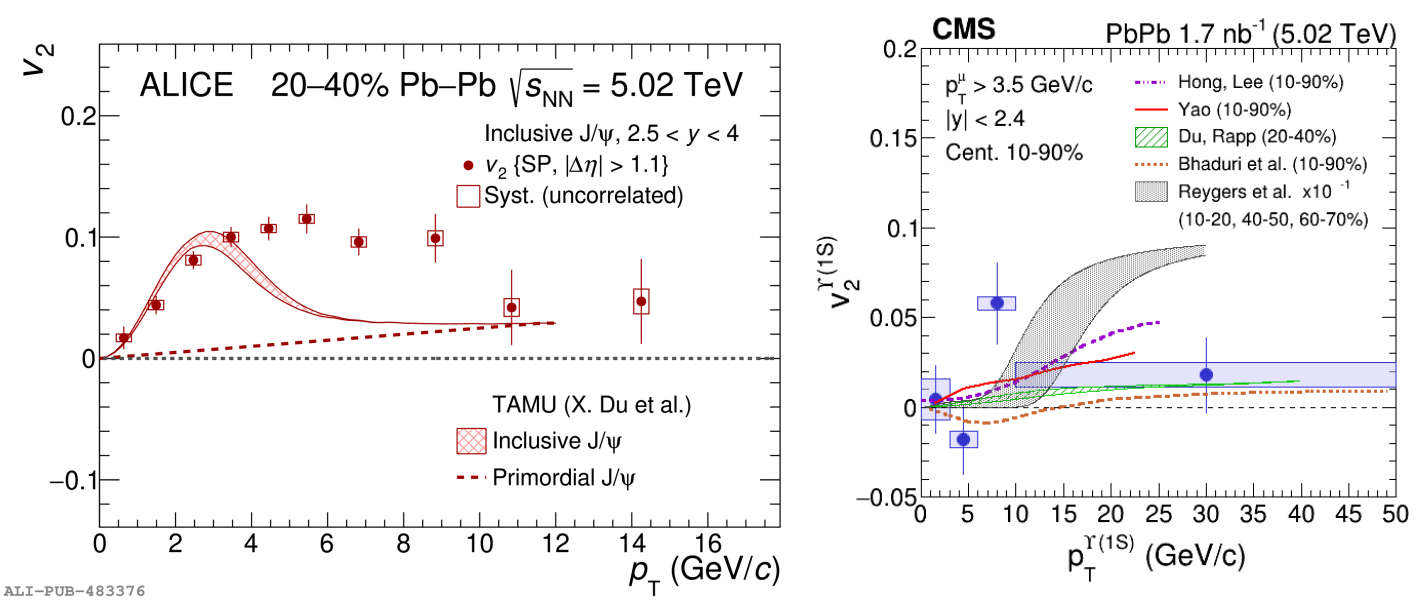

Figure 3: $v_{2}$ of $J / \psi[22]$ (left) and $\Upsilon(1 \mathrm{~S})$ [24] (right) as a function of $p_{\mathrm{T}}$.

of thermalization of charm quarks in the medium. The sizable $J / \psi v_{2}$ above $p_{\mathrm{T}} 4 \mathrm{GeV} / c$, deemed due to energy loss, is not yet fully understood, challenging theoretical developments. Completely different from $J / \psi$, no evidence of finite $\Upsilon(1 \mathrm{~S}) v_{2}$ was found in the currently studied kinematic range which is fairly described by several theoretical predictions [23, 24]. Based on the large b-quark mass shifting flow effect to large $p_{\mathrm{T}}$ [25], improved precision measurements especially at higher $p_{\mathrm{T}}$ will shed light on understanding the azimuthal anisotropy of $\Upsilon(1 \mathrm{~S})$. Complementary to $R_{\mathrm{AA}}$ and $v_{2}$, the polarization of quarkonium can provide information on its production modified in the presence of the QGP. First measurements of $J / \psi$ and $\Upsilon$ polarization in $\mathrm{Pb}-\mathrm{Pb}$ collisions at the LHC were carried out by the ALICE Collaboration [26]. The results indicate a small transverse polarization of $J / \psi$ in the helicity frame at low $p_{\mathrm{T}}$ while $\mathrm{LHCb}$ results in pp collisions showed small longitudinal polarization in a similar kinematic domain [27]

There are further possibilities to understand the QGP via studies of quarkonium production with new observables. The LHCb Collaboration found that prompt $J / \psi$ is produced with far more jet activity than in LO NRQCD-based predictions as implemented in PYTHIA for pp collisions via the measurement of prompt $J / \psi$ in jets [28]. Subsequent measurements and theoretical calculations suggest the production of charm quarks late in the parton shower [29, 30]. A similar study was extended to $\mathrm{Pb}-\mathrm{Pb}$ collisions by the CMS Collaboration showing less suppression for isolated $J / \psi$ than for $J / \psi$ with larger jet activity [31]. This result, as well as the high $p_{\mathrm{T}} J / \psi R_{\mathrm{AA}}$ which shows similar in shape and size as the one of charged particles and D-mesons [32], suggests high $p_{\mathrm{T}} J / \psi$ as a potential tool for studying gluon fragmentation and in medium energy loss mechanisms. In peripheral collisions, a large excess in the yield of $J / \psi$ mesons at low $p_{\mathrm{T}}\left(p_{\mathrm{T}}<\right.$ $300 \mathrm{MeV} / c$ ) with respect to expectations from purely hadronic production was observed by the ALICE Collaboration [33]. From further measurements and theoretical developments, coherent photonuclear production was suggested as the underlying physics mechanism [34, 35]. Prompt $J / \psi$ measurement in peripheral $\mathrm{Pb}-\mathrm{Pb}$ collisions from $\mathrm{LHCb}$ [36] strongly supported this hypothesis as the measured mean $p_{\mathrm{T}}$, constrained by the size of the nucleus target, is consistent with that expected for an $\mathrm{Pb}$ nucleus in UPC [37]. Since the coherent photo-production of $J / \psi$ is produced by a two gluon exchange at $\mathrm{LO}$ in $\mathrm{QCD}$, coherent photoproduction in inelastic $\mathrm{Pb}-\mathrm{Pb}$ collisions, will be a 
probe of the initial state in addition to the measurements in UPC giving both access to the gluons in the nucleus [38].

A wealth of quarkonium measurements, multi-differential and manifold, has been carried at the LHC by the ALICE, ATLAS, CMS and LHCb Collaborations in $\mathrm{pp}, \mathrm{p}-\mathrm{Pb}$ and $\mathrm{Pb}-\mathrm{Pb}$ collisions. The large data samples expected in the upcoming Run 3 with upgraded detectors, as well as in the high-luminosity phase of the collider for $\mathrm{Pb}-\mathrm{Pb}$ collisions, will enable the extension of the set of observables, provide improved precision measurements and lead to a quantitative understanding of the QGP $[39,40]$.

\section{References}

[1] ALICE Collaboration, Centrality and transverse momentum dependence of inclusive $J / \psi$ production at midrapidity in Pb-Pb collisions at $\sqrt{s_{\mathrm{NN}}}=5.02 \mathrm{TeV}$, Phys. Lett. B 805 (2020) 135434.

[2] R.L. Thews, M. Schroedter and J. Rafelski, Enhanced J/ $\psi$ production in deconfined quark matter, Phys. Rev. C 63 (2001) 054905.

[3] P. Braun-Munzinger and J. Stachel, (non)thermal aspects of charmonium production and a new look at J/4 suppression, Phys. Lett. B 490 (2000) 196.

[4] X. Du, M. He and R. Rapp, Color screening and regeneration of bottomonia in high-energy heavy-ion collisions, Phys. Rev. C 96 (2017) 054901.

[5] S. Digal, P. Petreczky and H. Satz, Quarkonium feed-down and sequential suppression, Phys. Rev. D 64 (2001) 094015.

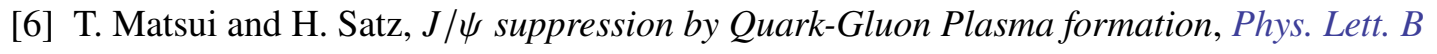
178 (1986) 416.

[7] A. Andronic, P. Braun-Munzinger, K. Redlich and J. Stachel, Decoding the phase structure of QCD via particle production at high energy, Nature 561 (2018) 321.

[8] E.G. Ferreiro and J.P. Lansberg, Is bottomonium suppression in proton-nucleus and nucleus-nucleus collisions at LHC energies due to the same effects?, JHEP 2018 (2018) 94.

[9] A. Rothkopf, Heavy quarkonium in extreme conditions, Phys. Rept. 858 (2020) 1.

[10] ALICE Collaboration, The ALICE experiment at the CERN LHC, JINST 3 (2008) S08002.

[11] ATLAS Collaboration, The ATLAS experiment at the CERN Large Hadron Collider, JINST 3 (2008) S08003.

[12] CMS Collaboration, The CMS Experiment at the CERN LHC, JINST 3 (2008) S08004.

[13] LHCb Collaboration, The LHCb detector at the LHC, JINST 3 (2008) S08005.

[14] ALICE Collaboration, Multiplicity dependence of $J / \psi$ production at midrapidity in $p p$ collisions at $\sqrt{s}=13 \mathrm{TeV}$, Phys. Lett. B $\mathbf{8 1 0}$ (2020) 135758. 
[15] J.-P. Lansberg, New observables in inclusive production of quarkonia, Phys. Rept. 889 (2020) 1 .

[16] S. Porteboeuf and R. Granier de Cassagnac, $J / \psi$ yield vs. multiplicity in proton-proton collisions at the LHC, Nucl. Phys. B-Proceedings Supplements 214 (2011) 181.

[17] CMS Collaboration, Investigation into the event-activity dependence of $\Upsilon(n S)$ relative production in proton-proton collisions at $\sqrt{s}=7$ TeV, JHEP 11 (2020) 001.

[18] E.G. Ferreiro, Excited charmonium suppression in proton-nucleus collisions as a consequence of comovers, Phys. Lett. B 749 (2015) 98.

[19] CMS Collaboration, Nuclear modification of $\Upsilon$ states in pPb collisions at $\sqrt{s_{\mathrm{NN}}}=5.02 \mathrm{TeV}$, CMS-PAS-HIN-18-005.

[20] ALICE Collaboration, Measurement of nuclear effects on $\psi(2 S)$ production in $p-P b$ collisions at $\sqrt{s_{\mathrm{NN}}}=8.16 \mathrm{TeV}$, JHEP 2020 (2020) 237.

[21] LHCb Collaboration, Measurement of the prompt-production cross-section ratio $\sigma\left(\chi_{c 2}\right) / \sigma\left(\chi_{c 1}\right)$ in pPb collisions at $\sqrt{s_{\mathrm{NN}}}=8.16 \mathrm{TeV}$, Phys. Rev. C 103 (2021) 064905.

[22] ALICE Collaboration, $J / \psi$ elliptic and triangular flow in $\mathrm{Pb}-\mathrm{Pb}$ collisions at $\sqrt{s_{\mathrm{NN}}}=5.02 \mathrm{TeV}, \mathrm{JHEP} 2020$ (2020) 141.

[23] ALICE Collaboration, Measurement of $\Upsilon(1 S)$ elliptic flow at forward rapidity in $\mathrm{Pb}-\mathrm{Pb}$ collisions at $\sqrt{s_{\mathrm{NN}}}=5.02$ TeV, Phys. Rev. Lett. 123 (2019) 192301.

[24] CMS Collaboration, Measurement of the azimuthal anisotropy of $\Upsilon(1 S)$ and $\Upsilon(2 S)$ mesons in PbPb collisions at $\sqrt{s_{\mathrm{NN}}}=5.02 \mathrm{TeV}$, Phys. Lett. B 819 (2021) 136385.

[25] K. Reygers, A. Schmah, A. Berdnikova and X. Sun, Blast-wave description of $\Upsilon$ elliptic flow at energies available at the CERN Large Hadron Collider, Phys. Rev. C 101 (2020) 064905.

[26] ALICE Collaboration, First measurement of quarkonium polarization in nuclear collisions at the LHC, Phys. Lett. B 815 (2021) 136146.

[27] LHCb Collaboration, Measurement of J/ $\psi$ polarization in pp collisions at $\sqrt{s}=7 \mathrm{TeV}$, Eur. Phys. J. C 73 (2013) 2631.

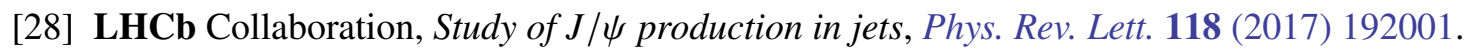

[29] R. Bain, Y. Makris, T. Mehen, L. Dai and A.K. Leibovich, NRQCD confronts LHCb data on quarkonium production within jets, Phys. Rev. Lett. 119 (2017) 032002.

[30] CMS Collaboration, Study of J/ $\psi$ meson production inside jets in pp collisions at $\sqrt{s}=8 \mathrm{TeV}$, Phys. Lett. B 804 (2020) 135409.

[31] CMS Collaboration, Fragmentation of jets containing a prompt $\mathrm{J} / \psi$ meson in $\mathrm{PbPb}$ and $p p$ collisions at $\sqrt{s_{\mathrm{NN}}}=5.02 \mathrm{TeV}$, arXiv:2106.13235. 
[32] ATLAS Collaboration, Prompt and non-prompt $J / \psi$ and $\psi(2 S)$ suppression at high transverse momentum in $5.02 \mathrm{TeV} P b+P b$ collisions with the ATLAS experiment, Eur. Phys. J. C 78 (2018) 762.

[33] ALICE Collaboration, Measurement of an excess in the yield of $J / \psi$ at very low $p_{T}$ in Pb-Pb collisions at $\sqrt{s}_{n n}=2.76 \mathrm{TeV}$, Phys. Rev. Lett. 116 (2016) 222301.

[34] STAR Collaboration, Observation of excess $J / \psi$ yield at very low transverse momenta in $\mathrm{Au}+\mathrm{Au}$ collisions at $\sqrt{s_{\mathrm{NN}}}=200 \mathrm{GeV}$ and $\mathrm{U}+\mathrm{U}$ collisions at $\sqrt{s_{\mathrm{NN}}}=193 \mathrm{GeV}$, Phys. Rev. Lett. 123 (2019) 132302.

[35] W. Zha, S.R. Klein, R. Ma, L. Ruan, T. Todoroki, Z. Tang et al., Coherent J/4 photoproduction in hadronic heavy-ion collisions, Phys. Rev. C 97 (2018) 044910.

[36] LHCb Collaboration, Study of $J / \psi$ photo-production in lead-lead peripheral collisions at $\sqrt{s_{\mathrm{NN}}}=5 \mathrm{TeV}$, arXiv:2108.02681.

[37] ALICE Collaboration, First measurement of the $|t|$-dependence of coherent $J / \psi$ photonuclear production, Phys. Lett. B 817 (2021) 136280.

[38] J.G. Contreras and J.D. Tapia Takaki, Ultra-peripheral heavy-ion collisions at the LHC, Int. J. Mod. Phys. A 30 (2015) 1542012.

[39] Z. Citron et al., Report from Working Group 5: Future physics opportunities for high-density QCD at the LHC with heavy-ion and proton beams, CERN Yellow Rep. Monogr. 7 (2019) 1159 .

[40] E. Chapon et al., Perspectives for quarkonium studies at the high-luminosity LHC, arXiv:2012.14161. 